\title{
ANÁLISIS DE UN MARCO: EL RAPTO (1965) DE FRANCISCO AYALA
}

\author{
Antonio Candeloro \\ Universidad Católica de Murcia \\ acandeloro@ucam.edu
}

Resumen: En este trabajo se analiza El rapto en relación con los intertextos que determinan su estructura y su significado. El objetivo es demostrar como la reescritura de algunos capítulos del Quijote cervantino le permite al autor llevar a cabo un discurso ideológico en el que se critican las posturas más conservadoras de la España franquista de los años 60. En este contexto, las referencias múltiples a Cervantes nos permiten reflexionar sobre el sexo, las relaciones entre hombres y mujeres, las relaciones entre España y el resto de países europeos.

Palabras clave: Francisco Ayala, novela corta, El rapto, intertextualidad, reescritura.

\section{ANALYSIS OF A FRAMEWORK: EL RAPTO (1965) BY FRANCISCO AYALA}

\begin{abstract}
In this work El rapto is analyzed in relation to the intertexts that determine its structure and its meaning. The objective is to demonstrate how the rewriting of some chapters of the Cervantine Quixote allows the author to carry out an ideological discourse in which the most conservative postures of the Francoist Spain of the 1960s are criticized. In this context, multiple references to Cervantes allow us to reflect on sex, relationships between men and women, relations between Spain and the rest of European countries.
\end{abstract}

Keywords: Francisco Ayala, nouvelle, El rapto, intertextuality, rewriting.

DOI: https://doi.org/10.24029/lejana.2020.13.428

Recibido: el 24 de noviembre de 2018

Aceptado: el 1 de marzo de 2019

Publicado: el 19 de febrero de 2020 


\section{Prólogo}

Como sabemos, además de su labor de escritor, Francisco Ayala ha desarrollado a lo largo de toda su vida una notable tarea de crítico literario y de teórico de la literatura (además de docente durante los años de su exilio, tanto en Hispanoamérica como en EE.UU.). ${ }^{1}$ En particular, ha profundizado a lo largo de los años en el ámbito de los estudios sobre algunos grandes clásicos de la literatura española como son Cervantes, Quevedo, Galdós y Unamuno. A Cervantes, en particular, Ayala le dedica la mayoría de sus esfuerzos interpretativos, como podemos comprobar en su recopilación La invención del Quijote. Indagaciones e investigaciones cervantinas $^{2}$ (la recopilación se publicó en el 2005, en coincidencia con el cuarto centenario de la aparición de la primera parte del Quijote). Y un homenaje a Cervantes es también El rapto, una novela corta que Ayala publica en el 1965 tras aceptar la invitación de Camilo José Cela para inaugurar la colección de la Novela Popular Española; ${ }^{3}$ no es casualidad que, al año siguiente, El rapto se convierta en el primer capítulo de la recopilación de narraciones breves De raptos, violaciones y otras inconveniencias, ${ }^{4}$ permitiendo al autor dar a la luz un conjunto que, a pesar de los diferentes tonos y estilos adoptados para cada pieza literaria, expresa una unidad de fondo en cuanto al contenido, siendo el sexo y la representación entre grotesca y escatológica de la realidad cotidiana uno de los elementos temáticos o ejes centrales alrededor de los cuales girarán los varios relatos.

El objetivo de este artículo es averiguar de qué forma el fenómeno intertextual influye en la escritura y en la articulación del discurso narrativo en El rapto y de qué forma el análisis de los intertextos (que la crítica, en su día, ya se preocupó en investigar) permiten leer e interpretar El rapto como, por un lado, un artilugio literario perfectamente armado para que el lector se involucre en la búsqueda del sentido a través de los mismos hipotextos citados o aludidos en la trama, y, por otro lado, como una obra literaria que mantiene con el lector un discurso enmascarado y sutil que lo obliga a posicionarse ante las reflexiones ideológicas que sugiere el autor detrás de la máscara de los dos narradores que cuentan los hechos (el narrador en primera persona que aparece en la "Introducción" y el narrador externo y en tercera persona que narra El rapto en cuanto nouvelle). ${ }^{5}$ Veremos cómo Cervantes deja su huella tanto en la "Introducción" como en la trama principal de la obra misma; y veremos cómo Ayala no se limita a re-escribir la narración de Eugenio, Leandra y Vicente de la Roca que aparece en el capítulo 51 de la I Parte del Quijote, ni tampoco a re-escribir (de forma transversal y originalísima, como ha demostrado la crítica ayaliana) la novela intercalada del Curioso impertinente, ${ }^{6}$ que ocupa los capítulos 33-35 de la I Parte de la obra cervantina, sino que, a la luz de la narrativa y de la poética del mismo Cervantes, Ayala desarrolla un discurso

\footnotetext{
${ }^{1}$ Para un acercamiento a las vicisitudes biográficas del escritor, cfr. Ayala, 2006.

${ }^{2}$ Cfr. Ayala, 2005.

${ }^{3}$ Para una reconstrucción del problema del género literario al que pertenece la obra, cfr. Pujante Segura, 2018.

${ }^{4}$ Cfr. Ayala, 1966 (todas las citas siguientes serán extrapoladas de esta edición).

${ }^{5}$ Sobre el primer aspecto, son fundamentales los siguientes estudios (que nos han ayudado a la hora de volver sobre los hipotextos mismos de los que se nutre la nouvelle): Ellis, 1969; García Montoro, 1968; Sánchez, 1966; Durán, 1977; Gruia, 2011; Pozuelo Yvancos, 2015.

${ }^{6}$ La primera en subrayarlo fue Escudero Martínez, 1989; Pozuelo Yvancos, en el artículo ya citado de 2015, volverá a remarcar las interconexiones entre El rapto y el Curioso impertinente, pero dejando de lado el tema que más nos ayudará a dar una nueva interpretación a partir del plano sexual y de las relaciones entre hombres y mujeres.
} 
ideológico y moral que tocará temas tan complejos como la percepción de España en los años 60 por parte de los exiliados, la percepción de las relaciones sentimentales entre hombres y mujeres y la percepción del sexo y de la homosexualidad masculina a partir, entre otras cosas, del análisis que ese mismo año (1965) Ayala le dedica al Curioso impertinente de Cervantes para iluminar la que él define como "una de las creaciones más ambiguas e insondables de su ambiguo e insondable autor" (Ayala, 2007b: 203). ${ }^{7}$ Se trata de una definición que bien podríamos aplicar a El rapto, como veremos en seguida.

\section{Análisis de la "Introducción" de El rapto (o de la importancia del marco)}

Empecemos por el principio. El rapto es una novela corta que, aun sin marco explícito, sí se presenta enmarcada por una "Introducción" de dieciséis páginas (en la edición de Alfaguara de 1966 a la que haremos referencia). Esto quiere decir que el lector no podrá obviar lo que se le cuenta en esa "Introducción" a la hora de abordar la interpretación del significado de la misma novela corta. Sin esta "Introducción", nos resultaría imposible o muy difícil entrar dentro del universo ficticio que nos presenta El rapto. ${ }^{8}$

Pues bien, lo que resulta más llamativo de la misma "Introducción" es precisamente la adopción de la primera persona de singular por parte del narrador que - como ya ha demostrado gran parte de la crítica ayaliana $-{ }^{9}$ coincide o podría coincidir con el mismo autor empírico, esto es, alguien que "A finales del año 1961" y "durante el mes de noviembre" viaja desde EE.UU. a la "histórica ciudad de Münster" para participar en un "congreso sobre el desarrollo económico-social de América Latina" (9). Está claro que solo el lector que disponga (hoy o en el 1965) de los datos biográficos reales de Ayala podría demostrar, efectivamente, el parecido íntimo (por no decir la identidad total) entre este "yo" y el autor de carne y hueso. En realidad, para los fines de la interpretación de lo que vendrá después —esto es, la novela corta en sí misma - no nos aporta nada (o nos aporta muy poco) el conocimiento de las barreras o de las fronteras reales entre uno y otro "yo"; porque Ayala adopta una máscara ${ }^{10}$ ya desde los umbrales del texto (por decirlo a la manera de Genette, 2001) y esto es fácil comprobarlo si leemos la primera frase que inaugura la misma "Introducción": "Mucho había oído ponderar yo, y mucho había leído también, acerca de la enorme afluencia de obreros españoles, atraídos y absorbidos por la industria de la nueva Alemania democrática (9; las cursivas son mías).

Se trata de un eco evidente de otra "Introducción", mucho más famosa: "Muchas veces tomé la pluma para escribille [el prólogo], y muchas la dejé, por no saber lo que escribiría" (Cervantes, 2015: 10). Es evidente que quien toma las riendas de la narración lo hace aludiendo al Quijote cervantino ya desde estas primerísimas palabras. Ayala juega a hablar y a

\footnotetext{
${ }^{7}$ Originalmente aparecido en Revista de Occidente, 30, 1965: 287-306.

${ }^{8}$ Lo mismo vale para el Lazarillo de Tormes: la lectura y la posterior interpretación de la narración cambiaría radicalmente si no apareciera ese "Prólogo" en el que Lázaro finge —o simula o pretende que pensemos— que le escribe una carta al famoso y anónimo "Vuesa Merced" que le pide que le cuente y relate detalladamente "el caso".

${ }^{9}$ El primero en notarlo fue Ellis (1969); Ayala aprovechará este análisis para profundizar desde el punto de vista teórico sobre la "ficcionalización" del autor dentro del universo de la narración (1990: 37-38).

${ }^{10} \mathrm{O}$ declina una "figuración" de su "yo", por retomar la feliz y útil expresión inventada por Pozuelo Yvancos, 2010.
} 
contar como Miguel de Cervantes para introducirnos en una especie de narración contemporánea (1965) a la luz de la narración clásica (1605) empezando ya desde el íncipit de la misma "Introducción" el juego intertextual que le compete al lector ideal saber reconocer. ${ }^{11}$ Si Cervantes nos confiesa que "muchas veces" intentó escribir el prólogo a su obra maestra (y que terminará escribiendo un prólogo sobre la imposibilidad de escribir los prólogos con la intervención milagrosa y teatral de su buen amigo "gracioso y bien entendido" (2015:11), Ayala adopta la máscara del narrador "al estilo cervantino" para confesarnos que "muchas veces" había oído hablar de los obreros españoles emigrados a Alemania, pero que nunca había tenido la oportunidad de hablarles en directo y en vivo: “¿Cómo, sin embargo, abordarlos y entablar con ellos una conversación que no resultara precaria, forzada, precipitada, extemporánea?” (9). Frente a esta cuestión surge otra pregunta: ¿de qué nace la necesidad del narrador de entablar conversación con los emigrados españoles? Si nos atenemos al texto y leemos la "Introducción" hasta el final, podríamos descubrir que es precisamente la condición de "exiliado" o de "emigrado" lo que une anímicamente al narrador (ese conferenciante, de origen granadino, que viene de EE.UU. para hablar de los problemas de Hispanoamérica en Alemania) a los obreros españoles, que, como muchos otros conterráneos, han tenido que dejar su país de origen para sobrevivir y para encontrar un trabajo digno o pagado dignamente.

Así que se trata de un problema de estricta y candente actualidad, si lo situamos en la España franquista de los años 60; pero el filtro con que el narrador (y detrás de él, el autor empírico) mira este problema es un filtro de tipo literario y cervantino. Igual de cervantina es la presentación de esos obreros en la estación de trenes en la que, finalmente, el narrador tendrá la oportunidad de charlar con ellos. La "Introducción" reproduce los diálogos entre el narrador y sus compatriotas durante toda la duración del viaje en tren desde Münster hasta Madrid, pasando por París (pero en la p. 21 también se cita la ciudad de Düsserdolf), lo que implica que la representación del espacio también es realista; el narrador quiere que nos ubiquemos tanto en el tiempo como en el espacio geográfico en el que ocurre el encuentro y la consiguiente conversación en tren. En este caso, además de cervantina, la narración a través del diálogo recuerda también otro género literario: las muchas novelas cortas que duran lo que dura un viaje. Si el modelo más antiguo puede ser considerada la obra de Geoffrey Chaucer (y sus Canterbury Tales), modelos más ligados a la Literatura del Siglo de Oro pueden ser las varias novelas intercaladas de autores de moda que imitan los rasgos de la literatura picaresca como son Alonso de Castillo Solórzano y Alonso Salas Jerónimo de Barbadillo: ambos añaden novelas cortas dentro de la trama principal de sus novelas picarescas para entretener el tiempo de los personajes que viajan siguiendo el patrón del "alivio para caminantes"; ambos utilizan el diálogo como elemento de arranque de esas mismas novelas cortas; ${ }^{12}$ y ambos

\footnotetext{
${ }^{11}$ Porque, como nos recuerda el mismo Ayala: "El arte de la composición organiza el material narrativo, es decir, presenta el argumento de manera óptima y con la máxima eficacia a fin de ofrecer una visión personal de los hechos y, con esto, dar una interpretación de ellos correspondiente a la visión del mundo propia del escritor. Esa visión constituye su originalidad de poeta, pero se transmite mediante sus destrezas de artista literario. Es el resultado de unas técnicas - en parte aprendidas de una tradición, en parte inventadas renovadoramente-, de una retórica aplicada a obtener la más adecuada expresión de un punto de vista" (1990: 121). Es evidente no solo en El rapto, sino en el resto de la obra narrativa de Ayala la huella de la tradición áurea y cervantina; Ayala construye y basa su originalidad y su punto de vista en los inventos del autor del Quijote.

${ }^{12}$ Dos ejemplos emblemáticos: Salas Barbadillo, La hija de la Celestina (1612-14); y Castillo Solórzano, La garduña de Sevilla (1642), en la que el autor introduce la novela corta "Quien todo lo quiere, todo lo pierde" que
} 
podrían estar detrás del "alivio para viajantes en tren" que Ayala escenifica en esta "Introducción".

Ahora bien, teniendo en cuenta que el narrador se configura como "figuración del yo" de Ayala según rasgos y tonos decididamente cervantinos, ¿de qué hablan realmente el narrador y los obreros españoles? Es importante intentar contestar esta pregunta porque como veremos - de aquí surgirán también los temas más importantes de El rapto.

Como ha notado y subrayado la crítica más atenta, esta "Introducción" aborda varios temas, siendo el tiempo uno de los elementos sobre los que más reflexiona el narrador (y, consecuentemente, sobre los que más se nos invita a reflexionar). La cita del lugar (Münster) y del año (1961) nos obliga a considerar la contemporaneidad casi perfecta entre el tiempo narrado y el tiempo de la narración (o entre el tiempo que evoca el narrador y el acto mismo de la escritura, esto es, el año 1965). Sin embargo, el pasado interviene inmediatamente para proyectar su sombra en el presente; es lo que ocurre en el principio de la "Introducción" y en el final, en dos momentos clave de toda la narración: "El burgomaestre de Münster nos había recibido en la sala donde hace más de tres siglos se concertó el Tratado de Westfalia, iluminada con candelabros como entonces. «Parecía que estuviéramos velando el cadáver del Imperio español», susurré en medio de tanta solemnidad a un colega venezolano que tenía a mi lado" (10).

En este caso, el presente relacionado con el acto institucional del congreso remite y retrotrae al narrador hacia un pasado histórico emblemático: el Tratado de Westfalia, aquí descrito como el acto de defunción de España en cuanto Imperio. Se trata, efectivamente, de un evento histórico luctuoso para la nación a la que pertenece el narrador (y los obreros con los que luego entablará el diálogo). Si Alemania se presenta como un país que es civilizado y que atrae a los jóvenes españoles que allí se trasladan para trabajar dignamente ("la nueva Alemania democrática" es como la define el narrador en la p. 9), España se presenta al mismo tiempo como país "no democrático" — si lo relacionamos con el año 1961 y la dictadura franquista - y "cadáver" — si lo relacionamos con el siglo XVII, cuando, efectivamente, el Imperio español cede ante las demás potencias de la Europa de 1640 y, con Felipe IV, empieza a vivir la fase del mayor declino económico y político tras su protagonismo de los siglos anteriores-.

La segunda escena se desarrolla en la parte conclusiva de la "Introducción": aludiendo a un modelo de coche alemán que estuvo en boga también en España durante los años de la guerra civil, y dándose cuenta de que sus interlocutores no lo entienden, el narrador toma consciencia del hecho de que les está hablando a unos jóvenes que no vivieron aquellos eventos trágicos: “Antes de la guerra andaban por España muchísimos, del viejo modelo; pero ustedes, claro no se acordarán. El salmantino, con su sonrisa desamparada y cómica, contesta: - ¡Cómo voy a acordarme, si no había nacido!- (24). La respuesta alegre y directa, espontánea e inocente del joven empuja al narrador a una nueva reflexión sobre el paso del tiempo y sobre la reconstrucción cronológica de los hechos pasados: "Y yo pienso: «La guerra de España pertenece a la historia, ya. Este mismo año se han publicado dos o tres historias de

dura lo que dura el viaje: "aquí tuvo fin la novela, que duró hasta que llegaron al fin de la jornada de aquel día" (1972: 100). Sobre esta técnica cfr. Baquero Escudero, 2013; Rey Hazas, 2003. 
la guerra civil española. Y estos muchachos que trabajan en la industria de la nueva Alemania, todavía no habían nacido»" (24).

La consideración, de tono pesimista, abre un abismo temporal sobre la misma percepción del tiempo histórico: el hecho de que en 1961 se hayan publicado ya "dos o tres historias de la guerra civil" nos obliga a reflexionar, en cuanto testigos no directos de esos eventos trágicos, sobre la facilidad con que el pasado se convierte en Historia cuando median tan solo veintidós años entre los "eventos reales" y los "eventos narrados". Es como si Ayala se sorprendiera él mismo al comprobar que la guerra civil es ya parte de la Historia y está ya narrada dentro de varios ensayos historiográficos (e incluso podríamos ponerles un título a esas "dos o tres historias de la guerra civil" a las que alude el narrador). Es como si resultara absurdo o raro que eventos todavía vivos en la memoria de los supervivientes (como Ayala, exiliado) formaran ya parte de la Historia redactada por los demás. Al mismo modo resulta extraño y perturbador constatar que esos jóvenes españoles que trabajan en Alemania todavía no habían nacido cuando sucedió aquello. La pregunta que surge a la luz de estas observaciones es la siguiente: ¿volverá a aparecer el tema - todavía candente- del recuerdo de la reciente guerra civil española en El rapto? ¿O es que el autor, a través de la máscara del narrador, se limita a evocar estos eventos luctuosos relacionados con España tan solo en la “Introducción”?

Volvamos al texto: hay otro tema que el narrador subraya y tiene que ver con las relaciones entre hombres y mujeres y las diferencias de las relaciones de pareja entre España y Alemania. Hablando de las dificultades del idioma, uno de los españoles confiesa al narrador que las chicas alemanas les ayudan y "tienen bastante paciencia" (13). De hecho, el narrador se fijará en una rubia que acompaña al otro obrero sevillano con el que compartirá el viaje en tren. Estas son las palabras de alabanza del español hacia las alemanas: "-Las muchachas aquí - prosiguió - piensan que pueden hacer lo que les dé la gana, como los hombres; y tienen razón. Nadie las mira mal por eso; no es como en España. Después de todo, tienen razón, ¿no le parece a usted?” (13). Si en la "nueva Alemania democrática” las mujeres actúan "como los hombres" (con total libertad), en España, evidentemente, se vive un atraso importante y la sociedad machista impide, simplemente, que las mujeres tengan las mismas libertades que los hombres. Será un madrileño que se une al grupo de los obreros el encargado de ahondar en la comparación España-Alemania a partir de las relaciones sentimentales:

- Lo que ocurre es que los alemanes tienen casa; y si un hombre está casado, y contento con su mujer, ¿qué mejor cuando termina el trabajo, que irse a ver la televisión en casa, muy cómodo, y con su cervecita al lado? No es porque sean alemanes; todo el mundo haría igual, si pudiera. En cambio, ¿qué es lo que pasa en España? Vuelve el hombre reventado de cinchar, y no se encuentra más que problemas y malos humores; que si hay que pagar esto, que si los zapatos, que no aguantan más, que... Total, se sale para la taberna de la esquina, pide un litro de tinto, y se pone a hablar de fútbol. En España, lo único que se hace es hablar de fútbol. ¿O no tengo razón? (21-22; las cursivas son mías)

Es un fragmento importante: el madrileño le achaca el malestar general entre hombres y mujeres a la falta de dinero y de trabajo, y el vino y el fútbol se nos presentan aquí como las dos únicas válvulas de escape de una realidad tremendamente triste y pobre (a nivel material, pero también espiritual). Las mujeres españolas no solo no tienen libertad, como sí las alemanas, ni la independencia, sino que forman parte de la crisis existencial que viven 
muchos hombres que no encuentran salida a una crisis que es, sobre todo, económica. El tema sentimental se liga al tema económico ya en la presentación de los obreros españoles: la descripción que nos da el narrador vehicula la fascinación que sienten los emigrados hacia la ropa que pueden poseer trabajando fuera de España: “jóvenes vivaces, alegres, de pantalones estrechísimos a la moda italiana y esos chaquetones de gusto alemán, tan exagerados en su opulencia que seducen la fantasía de quienes nunca poseyeron nada; jóvenes de manos inquietas y voladoras dentro de sus enormes guantes" (23; las cursivas son mías). El contraste es evidente. Es más: en este fragmento, Alemania e Italia no se presentan solo como países libres (en cuanto democráticos), sino también como países símbolo del lujo, de la moda más llamativa, la que captura la fantasía de los demás españoles que, por ser tales, y viviendo en España, nunca podrían vestirse como los jóvenes de Alemania o de Italia. El elemento relacionado con la ropa y los objetos de lujo (o considerados tales por los demás) y el elemento sexual relacionado con la manera en la que interactúan hombres y mujeres en España serán básicos para la correcta interpretación de El rapto.

Manteniendo abierta la pregunta: ¿habla de la guerra civil esta novela corta?, nos vamos acercando al texto narrativo, teniendo en cuenta dos elementos también fundamentales: el primero, que la sombra alargada de Cervantes en la obra de Ayala empieza a asomar ya en la primera frase de la "Introducción"; el segundo, que el deseo al final cumplido de poder hablar con los jóvenes obreros españoles expatriados lleva a una contemplación amarga y a una reflexión pesimista sobre España: el "me duele España" unamuniano se convierte aquí en el ritornello de los obreros que exclaman: “QQué buen país sería España, si se pudiera vivir alli!” (18), para luego ser repetido con variación: “¿Qué buen país sería España, si uno pudiera vivir!" (19; en ambos casos, las cursivas son mías). El diálogo entre exiliados conduce al conocimiento del dolor por un país que se ve como inicuo o ya cadáver (desde el Tratado de Westfalia hasta la dictadura franquista todavía vigente en el 1961).

\section{Bibliografía}

AYALA, Francisco (1966): De raptos, violaciones y demás inconveniencias. Madrid, Alfaguara.

--- (1990): El escritor en su siglo. Madrid, Alianza.

--- (2005): La invención del Quijote. Indagaciones e invenciones cervantinas. Madrid, Suma de Letras.

--- (2006): Recuerdos y olvidos (1906-2006). Madrid, Alianza.

--- (2007b): "Los dos amigos". En Carolyn Richmond (ed.), Estudios literarios. Obras completas III. Barcelona, Círculo de Lectores / Galaxia Gutenberg: 647-672. [1989].

BAQUERO ESCUDERO, Ana L. (2013): La intercalación de historias en la narrativa de Cervantes. Pontevedra, Academia del Hispanismo.

CASTILlO SOLÓRZANO, Alonso de (1972): La garduña de Sevilla. Ed. Federico Ruiz Morcuende. Madrid, Espasa-Calpe.

CERVANTES, Miguel de (2015): Don Quijote de la Mancha. Ed. Instituto Cervantes, dir. Francisco Rico. Madrid, Espasa-Calpe. Barcelona, Círculo de Lectores. 
DURÁN, Manuel (1977): "Notas sobre Francisco Ayala, «El rapto», y el mito del eterno retorno". Cuadernos Hispanoamericanos 329/330 (1977): 441-448.

ELLIS, Keith (1969): “Cervantes and Ayala's «El Rapto»: The Art of Reworking a Story". PMLA 84, 1 (1969): 14-19. DOI: https://doi.org/10.2307/1261152

ESCUDERO MARTÍNEZ, Carmen (1989): Cervantes en la narrativa de Francisco Ayala. Murcia, Universidad de Murcia.

GARCÍA MONTORO, Adrián (1968): “El rapto, novela ejemplar”. La Torre: Revista de la Universidad de Puerto Rico 62 (1968): 151-165.

GENETTE, Gérard (2001): Umbrales. Buenos Aires, Siglo XXI.

GRUIA, Ioana (2011): "La seducción del cuento: huellas cervantinas en «El rapto» de Francisco Ayala”. En Luis García Montero y Milena Rodríguez Gutiérrez (eds.), De este mundo y los otros. Estudios sobre Francisco Ayala. Madrid, Visor Libros: 331340 .

POZUELO YVANCOS, José María (2010): Figuraciones del yo en la narrativa: Javier Marías y Enrique Vila-Matas. Valladolid, Universidad de Valladolid, Cátedra Miguel Delibes.

--- (2015): “Ayala y el Quijote: Lectura de «El rapto»”. En José María Pozuelo Yvancos et al. (coords.), De Re Poetica: homenaje al profesor D. Manuel Martínez Arnaldos. Murcia, Universidad de Murcia: 560-569.

PUJANTE SEGURA, Carmen M." (2018): "Francisco Ayala, escritor de novelas cortas: recontextualización y revalorización de El rapto". Lejana 11 (2018): 70-82. DOI: https://doi.org/10.24029/lejana.2018.11.257

REY HAZAS, Antonio (2003): Deslindes de la novela picaresca. Málaga, Universidad de Málaga.

SÁNCHEZ, Alberto (1996): “Cervantes y Francisco Ayala: original refundición de un cuento narrado en «El Quijote»”. Cuadernos Hispanoamericanos 196 (1966): 133-139.

(C) Antonio Candeloro

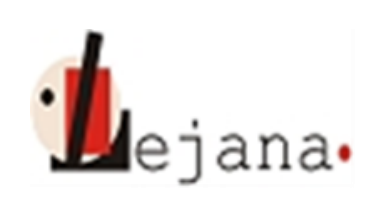

$\underline{\text { http://ojs.elte.hu/index.php/lejana }}$

Universidad Eötvös Loránd, Departamento de Español, 1088 Budapest, Múzeum krt. 4/C 